\title{
Height predict incident non-alcoholic fatty liver disease in a general adult population, independent of body mass index and metabolic syndrome
}

\section{Shubham Kumari}

Tianjin Medical University

\section{Xuena Wang}

Tianjin Medical University

\section{Yeqing Gu}

Tianjin Medical University

\section{Yuhan Huang}

Tianjin Medical University

\section{Qing Zhang}

Tianjin Medical University General Hospital

\section{Li Liu}

Tianjin Medical University General Hospital

\section{Ge Meng}

Tianjin Medical University

\section{Hongmei Wu}

Tianjin Medical University

\section{Shaomei Sun}

Tianjin Medical University General Hospital

\section{Xing Wang}

Tianjin Medical University General Hospital

\section{Ming Zhou}

Tianjin Medical University General Hospital

Qiyu Jia

Tianjin Medical University General Hospital

\section{Guolin Wang}

Tianjin Medical University General Hospital

\section{Kun Song}

Tianjin Medical University General Hospital

\section{Kaijun Niu ( $\nabla$ nkj0809@gmail.com )}

Tianjin Medical University 
Research article

Keywords: Early life experiences, height, insulin-like growth factor-1, non-alcoholic fatty liver disease Posted Date: November 8th, 2019

DOI: https://doi.org/10.21203/rs.2.17009/v1

License: (c) (i) This work is licensed under a Creative Commons Attribution 4.0 International License. Read Full License 


\section{Abstract}

Background Early-life hormonal and nutritional factors can greatly influence the risk of non-alcoholic fatty liver disease (NAFLD). Adult height is a simple marker for these factors. We aimed to evaluate adult height as a predictor of NAFLD.

Methods We performed a prospective cohort study of 35, 994 participants recruited from 2010 to 2016 in Tianjin, China. NAFLD was diagnosed by ultrasound. Adjusted Cox proportional hazards regression models were conducted in 2019 to assess the gender-specific association between the quintiles of height and the incidence of NAFLD.

Results Participants were followed up for 5.5 years with a mean follow-up of 2.6 years. During the followup period, 6,245 of 35,994 participants developed NAFLD. The multiple-adjusted hazard ratios (95\% confidence interval) of NAFLD for increasing quintiles of height were 1.00 (reference), $0.90(0.81,0.99)$, $0.97(0.87,1.07), 0.86(0.78,0.96)$, and $0.84(0.75,0.94)$ ( $P$ for trend $<0.01)$ in males and 1.00 (reference), $0.97(0.86,1.09), 0.98(0.86,1.11), 0.93(0.81,1.06)$, and $0.84(0.73,0.96)(P$ for trend $=0.02)$ in females, respectively.

Conclusions The study is the first to demonstrate adult height is a new, simple and inexpensive marker for ultra-early prediction of NAFLD.

\section{Background}

Non-alcoholic fatty liver disease (NAFLD) is defined as the presence of $\geq 5 \%$ of hepatic steatosis, in the absence of secondary causes of hepatic fat accumulation, such as chronic use of medications or significant alcohol intake(1). It is the most common chronic liver disease all over the world and its prevalence is constantly increasing(2). About $25 \%$ of world population was estimated to have NAFLD(3). Unhealthy lifestyles and dietary habits in addition to genetic predisposition have pose increased the prevalence of NAFLD in the Asia Pacific region(4).

Early life experiences, including nutrition and hormone, play an important role in influencing later susceptibility to chronic diseases by epigenetic mechanisms $(5,6)$. Accumulating evidence suggests that hormonal and nutritional experiences in early life may predispose high incidence of type 2 diabetes mellitus (T2DM) and insulin resistance in later life(7). Furthermore, there is sufficient evidence to demonstrate that T2DM, insulin resistance and NAFLD share many important metabolic risk factors and common pathogenetic mechanism(8). Several studies preliminarily suggested that growth hormone (GH) levels and insulin-like growth factor-1 (IGF-1) levels are negatively associated with NAFLD in adults(9, 10), and GH replacement therapy in GH-deficient patients can alleviate NAFLD and improve liver fibrosis(11). Moreover, suboptimal early life nutrition may increase the susceptibility, age on set, and severity of NAFLD(12). 
Adult height is defined as the tallest height after height velocity had decreased to $1 \mathrm{~cm}$ or less over 6 months and relatively fixed as compared with child or youth height(13). Adult height greatly reflects differences in nutrition and hormone levels in early life $(14,15)$. It is well recognized that GH and IGF-1 are strongly and positively associated with growth in height, and GH therapy in children with short stature caused by several diseases augments adult height(16). Since early hormonal and nutritional experiences are major risk factors of final height, it is assumed that adult height may be a potentially useful predictor of incident NAFLD. To date, few studies have focused on the association between adult height and NAFLD.

In the present study, we designed a prospective cohort study to determine whether adult height was associated with the risk of NAFLD in the general Chinese adult population.

\section{Methods}

\section{Study design and participants}

Details of the Tianjin Chronic Low-Grade Systemic Inflammation and Health (TCLSIH) Cohort Study have been described elsewhere(17). Briefly, participants were randomly recruited between January 2010 and December 2016 from the general population in Tianjin, China. All participants received annual health examinations (including liver ultrasound examination, anthropometric measurements, and blood tests) and completed a structured questionnaire survey. Written informed consent was obtained from all participants. The protocol of this study was approved by the Institutional Review Board of Tianjin Medical University.

From 2010 to 2016, a total of 90,536 participants received health examinations. We excluded 170 participants who had missing data on alanine aminotransferase, 4,013 participants had excessive alcohol intake (>140 g/week in males and $>70 \mathrm{~g} /$ week in females), and 28,935 participants who had NAFLD at baseline. Moreover, we excluded 767 participants with other liver diseases (including autoimmune liver diseases, chronic hepatitis B or C, cirrhotic or operation on liver), and those with a history of cardiovascular disease $(n=5,475)$ or cancer $(n=1,039)$, and those aged $<25$ years $(n=3,996)$. Furthermore, participants were also excluded if they were recruited in $2016(n=4,894)$ or were lost in following up ( $n=5,253)$. Finally, a total of 35,994 participants were available for analysis (follow-up rate: $87 \%$; followed up for 1-5.5 y; mean duration of follow-up (standard deviation): 2.6 (1.6)).

\section{Assessment of height}

Height was measured to the nearest $0.1 \mathrm{~cm}$ using a standard protocol. In order to investigate how height level is associated with NAFLD, we divided males and female participants into 5 categories (quintiles) according to height level (cm, range) as follows: (1) Level 1 (148.5-167.7), Level 2 (167.8-171.2), Level 3 (171.3-174.3), Level 4 (174.4-178.1), and Level 5 (178.2-204.1) in males; (2) Level 1 (138.0-156.2), 
Level 2 (156.3-159.5), Level 3 (159.6-162.1), Level 4 (162.2-165.4), and Level 5 (165.5-184.6) in females.

\section{Diagnosis of NAFLD}

Real-time ultrasonography performed by trained and certified technicians was used to diagnose NAFLD. Participants were considered to have NAFLD if (1) they had a self-reported alcohol intake of $<140 \mathrm{~g} /$ week and $<70 \mathrm{~g} /$ week for males and females, respectively; (2) and at least two of the following abnormal findings of abdominal ultrasound images: diffusely increased liver near field ultrasound echo; increased liver echotexture, compared to the kidneys; vascular blurring and the gradual attenuation of far field ultrasound echo(18).

\section{Assessment of other variables}

Waist circumference (WC) was measured using a nonelastic plastic anthropometric tape at the level of umbilicus with subjects standing and breathing normally.Participants rest for at least 5 minutes in a seated position prior to blood pressure measurements. Blood pressure was measured twice from participants' upper right arms using the TM-2655 device (A\&D Company Ltd, Tokyo, Japan), and the blood pressure value was recorded in average. Fasting blood samples for the analysis of biochemical values were collected in siliconized vacuum plastic tubes. Fasting blood glucose, total cholesterol, triglycerides, low-density lipoprotein cholesterol, and high-density lipoprotein cholesterol were measured using appropriate kits on a Cobas 8000 analyzer (Roche, Mannheim, Germany). We defined metabolic syndrome (MetS) according to the American Heart Association scientific statements of 2009(19). Alanine aminotransferase was measured by IFCC method.

Body weight was measured by training nurses, with participants without heavy clothes. Body mass index (BMI) was calculated as weight $(\mathrm{kg})$ divided by squared height $\left(\mathrm{m}^{2}\right)$. Information on family history of cardiovascular disease, family history of hypertension, family history of hyperlipidemia, and history of diabetes, was assessed at baseline using a structured questionnaire.

\section{Statistical analysis}

Baseline characteristics of participants were compared using analysis of variance for continuous variables and logistic regression analysis for categorical variables. Continuous variables were shown as geometric mean (95\% confidence interval $(\mathrm{Cl})$ ), and categorical variables were presented as percentage.

Because the interaction between sex and height was statistically significant $(P<0.0001)$, we analyzed the association between height and NAFLD stratified by sex. We fitted four Cox proportional hazards regression models to evaluate the association between baseline height and incident NAFLD. The initial model was unadjusted model (crude model). Model 2 was adjusted for age and WC. In model 3, we 
additionally adjusted for smoking status, alcohol drinking status, Mets, family history of cardiovascular disease, family history of family history of hypertension, family history of hyperlipidemia, and family history of diabetes. In model 4 , we further adjusted for baseline BMI. All $P$ values for linear trends were calculated using the median value for each quintile.

All statistical analyses were performed using SAS version 9.4 (SAS Institute, Inc.). Two-tailed $P<0.05$ was considered as statistically significant.

\section{Results}

In this study, $41.3 \%$ of participants were males and $58.7 \%$ were females. Mean age (standard deviation) years was 42.2 (12.9) in males and 39.2 (11.3) in females, respectively. During the 5.5 years follow-up period between 2010 and 2016, 6,245 of 35,994 individuals (17.4\%) developed NAFLD. The incidence of NAFLD was 65.8 per 1,000 person-years. In the 5 height quintiles, the respective rates of NAFLD were $110.1,104.4,113.2,108.7$ and 110.2 per 1000 person-years in males and 48.4, 43.1, 40.7, 35.9 and 31.7 per 1,000 person-years in females.

Characteristics of participants relative to NAFLD status for follow-up analysis are presented in Table 1. The mean age $(95 \% \mathrm{Cl})$ in non-NAFLD and NAFLD participants was $38.3(38.2,38.4)$ years and $41.4(41.1$, 41.7) years, respectively. Compared with participants without NAFLD, participants with NAFLD had older age, lower high-density lipoprotein cholesterol, but higher BMI, WC, total cholesterol, low-density lipoprotein cholesterol, triglycerides, fasting blood glucose, systolic blood pressure, diastolic blood pressure, and alanine aminotransferase (all $P<0.0001$ ). Participants with NAFLD tended to be male, smoker, ex-smoker, everyday drinker, sometimes drinker, while those without NAFLD tended to be nonsmoker and non-drinker (all $P<0.0001$ ). In addition, a higher proportion of participants with NAFLD had MetS and a family history of cardiovascular disease, hypertension and diabetes (all $P<0.0001$ ).

Table 2 and Table 3 show the crude and adjusted associations between quintiles of height and NAFLD in male and female participants, respectively. In the third multivariate model which didn't adjust for BMI, the adjusted HRs $(95 \% \mathrm{Cl})$ for NAFLD across height quintiles were 1.00 (reference), $0.86(0.78,0.95), 0.90$ $(0.81,0.99), 0.77(0.70,0.85)$ and $0.71(0.64,0.78)$ in males and 1.00 (reference), $0.92(0.82,1.04), 0.85$ $(0.75,0.97), 0.78(0.68,0.89)$ and $0.65(0.57,0.74)$ in females (both $P$ for trend $<0.0001)$. Similarly, in the final multivariate model which adjusted BMI, the adjusted HRs $(95 \% \mathrm{Cl})$ for NAFLD across height quintiles were 1.00 (reference), $0.90(0.81,0.99), 0.97(0.87,1.07), 0.86(0.78,0.96)$ and $0.84(0.75,0.94)$ in males ( $P$ for trend $<0.01)$ and 1.00 (reference), $0.97(0.86,1.09), 0.98(0.86,1.11), 0.93(0.81,1.06)$ and 0.84 $(0.73,0.96)$ in females $(P$ for trend $=0.02)$.

\section{Discussion}

In this large-scale prospective cohort study, we found that higher level of adult height was inversely associated with the risk of NAFLD among males and females in China. The inverse association remained 
even after controlling for potential confounding factors. To our knowledge, this is the first study to investigate the association between adult height and NAFLD.

We adjusted for multiple potentially confounding factors in our analysis. This study indicated that numerous factors (such as age, BMI, WC, smoking status, metabolic syndrome and family history of some diseases) correlated positively with NAFLD. We used crude model (model 1) first and results showed negative association between height and NAFLD in both males and females. It is well-recognized that NAFLD and height are related to age and $W C(20,21)$, so we adjusted for these two variables in model 2. Adjustment for age and WC made the associations in males more obvious compared with model 1 ; however, in females, this adjustment didn't significantly influence the associations in model 1, leading us to conclude that age and WC are major confounding factors in males but not in females. Since NAFLD was associated with WC, smoking status, drinking status, metabolic syndrome, family history of cardiovascular disease, hypertension, hyperlipidemia and diabetes(22, 23), we subsequently adjusted for these variables in model 3. After adjustments for these factors, the associations didn't change significantly in both males and females, implying these factors may not confound the association between height and NAFLD. In model 4, the present study adjusted BMI and variables in model 3 to confirm the role of BMI in association between height and NAFLD. This adjustment made the association less obvious in both males and females, suggesting that BMI play an important role in association between height and NAFLD.

To date, no studies have investigated the association between height and NAFLD. Several studies investigated the association between height and T2DM. Reports about the risk of T2DM and height have produced conflicting results $(24,25)$. A meta-analysis showed negative association between height and risk of T2DM in woman only(26), whereas a cohort study of Finnish men showed adult height is associated with decreased risk of T2DM (1). Since T2DM and NAFLD both result from metabolic dysregulation, these results are, to some extent, consistent with our novel findings that shorter people were associated with higher incidence of NAFLD. Compared to previous cohort studies using Chinese adults with overall incidence of NAFLD ranging from $15.2 \%$ to $24.8 \%(27)$, the overall incidence of NAFLD in our study is $17.4 \%$, consistent with previous studies.

The mechanisms of association between adult height and NAFLD may involve epigenetic changes induced by early life adversity. Interacting with epigenetic mechanisms, hormonal and nutritional conditions in early life influence both attained height and later susceptibility to NAFLD. GH and IGF-1 play essential roles in linear growth as well as in the liver metabolism(28). Therefore, epigenetic modification of IGF-1 gene might play a critical role in the development of NAFLD and the growth in height. NAFLD-specific expression and methylation differences between healthy controls and morbidly obese patients with all stages of NAFLD were seen for the IGF-1 gene, one of the key drivers of the liver's phenotype(29). Furthermore, the methylation of the IGF-1 P2 promoter showed a strong negative association with serum IGF-1 levels and height as a child(30). In addition, data from animal models have indicated that nutritional perturbation of epigenetic regulation is a likely link between prenatal and early postnatal nutrition and health status in later life(31). A study in mice showed that exposure to 
prenatal and post-weaning western-style diet predisposed male mouse offspring to the development of NAFLD in adulthood and induced alterations in DNA methylation in key metabolic genes(32). On the other hand, a recent study has demonstrated that height plays an important partial role in determining several aspects of a person's socioeconomic status(33). Since lower socioeconomic status is closely associated with increased levels of health impairment(34), socioeconomic status maybe an explanation for the association between height and NAFLD. Further research is warranted to validate the hypotheses.

The present study has several limitations. Firstly, NAFLD was diagnosed by abdominal ultrasound rather than liver biopsy, which is the gold standard for diagnosis of NAFLD. However, abdominal ultrasound is not invasive and widely used in large-scale population-based studies. Moreover, this noninvasive method has a sensitivity of $89 \%$ and a specificity of $93 \%(35)$. Second, although we adjusted for a considerable number of potential confounding factors in the present study, residual confounding cannot be excluded.

\section{Conclusions}

Adult height was negatively associated with incident NAFLD in males and female, independent of BMI and MetS. The present results indicate that adult height may be a useful predictor for NAFLD to identify high-risk populations and prevent NAFLD at an early age. Furthermore, this study provides clues to the mechanism of the link between early life experiences and NAFLD. Future studies are needed to elucidate the mechanism of association between adult height and the risk of NAFLD.

\section{List Of Abbreviations}

NAFLDNon-alcoholic fatty liver disease

T2DMType 2 diabetes mellitus

GHGrowth hormone

IGF-1 Insulin-like growth factor-1

WCWaist circumference

TCLSIHTianjin Chronic Low-Grade Systemic Inflammation and Health

MetS Metabolic syndrome

BMIBody mass index

ClConfidence interval

HRHazard ratio

\section{Declarations}




\section{Ethics approval and consent to participate}

The protocol of this study was approved by the Institutional Review Board of the Tianjin Medical University and each participant gave written informed consent prior to participation in the study.

\section{Consent for publication}

Not applicable

\section{Availability of data and materials}

The datasets generated and analysed during the current study are not publicly available due [public availability would compromise participant privacy] but are available from the corresponding author on reasonable request.

\section{Competing interests}

The authors declare that they have no competing interests.

\section{Funding}

This work was supported by grants from the National Natural Science Foundation of China (No. $81673166,81372118,81372467$ and 81302422$)$, China. The funder of the study had no role in study design, data collection, data analysis, data interpretation, or writing of the report.

\section{Authors' contributions}

Conception and design of the study: S.K., X.W., and K.N. Data collection and analysis: S.K., X.W., Y.G., Y.H., Q.Z., L.L., G.M., H.W., S.S., X.W., M.Z., Q.J., G.W., K.S., and K.N. Interpretation of data: S.K., X.W., and K.N. Drafting the manuscript: S.K., X.W., and K.N. All authors read and approved the final manuscript.

\section{Acknowledgements}

We gratefully acknowledge all of the people who participated in the study and Tianjin Medical University General Hospital-Health Management Centre for creating the possibility to perform the study.

\section{References}

1.Anstee QM, McPherson S, Day CP. How big a problem is non-alcoholic fatty liver disease? BMJ. 2011;343:d3897.

2. Woo Baidal JA, Lavine JE. The intersection of nonalcoholic fatty liver disease and obesity. Science translational medicine. 2016;8(323):323rv1. 
3.Younossi ZM, Koenig AB, Abdelatif D, Fazel Y, Henry L, Wymer M. Global epidemiology of nonalcoholic fatty liver disease-Meta-analytic assessment of prevalence, incidence, and outcomes. Hepatology (Baltimore, Md). 2016;64(1):73-84.

4.Duseja A, Chalasani N. Epidemiology and risk factors of nonalcoholic fatty liver disease (NAFLD). Hepatology international. 2013;7 Suppl 2:755-64.

5.Waterland RA, Jirtle RL. Early nutrition, epigenetic changes at transposons and imprinted genes, and enhanced susceptibility to adult chronic diseases. Nutrition (Burbank, Los Angeles County, Calif). 2004;20(1):63-8.

6.Wang G, Walker SO, Hong X, Bartell TR, Wang X. Epigenetics and early life origins of chronic noncommunicable diseases. The Journal of adolescent health: official publication of the Society for Adolescent Medicine. 2013;52(2 Suppl 2):S14-21.

7.Jiang X, Ma H, Wang Y, Liu Y. Early life factors and type 2 diabetes mellitus. Journal of diabetes research. 2013;2013:485082.

8.Bugianesi E, Moscatiello S, Ciaravella MF, Marchesini G. Insulin resistance in nonalcoholic fatty liver disease. Current pharmaceutical design. 2010;16(17):1941-51.

9.Xu L, Xu C, Yu C, Miao M, Zhang X, Zhu Z, et al. Association between serum growth hormone levels and nonalcoholic fatty liver disease: a cross-sectional study. PloS one. 2012;7(8):e44136.

10.Arturi F, Succurro E, Procopio C, Pedace E, Mannino GC, Lugara M, et al. Nonalcoholic fatty liver disease is associated with low circulating levels of insulin-like growth factor-I. The Journal of clinical endocrinology and metabolism. 2011;96(10):E1640-4.

11.Nishizawa H, Iguchi G, Murawaki A, Fukuoka H, Hayashi Y, Kaji H, et al. Nonalcoholic fatty liver disease in adult hypopituitary patients with $\mathrm{GH}$ deficiency and the impact of $\mathrm{GH}$ replacement therapy. European journal of endocrinology. 2012;167(1):67-74.

12.Li M, Reynolds CM, Segovia SA, Gray C, Vickers MH. Developmental Programming of Nonalcoholic Fatty Liver Disease: The Effect of Early Life Nutrition on Susceptibility and Disease Severity in Later Life. BioMed research international. 2015;2015:437107.

13.Taback SP, Collu R, Deal CL, Guyda HJ, Salisbury S, Dean HJ, et al. Does growth-hormone supplementation affect adult height in Turner's syndrome? Lancet (London, England). 1996;348(9019):25-7.

14.Silventoinen K. Determinants of variation in adult body height. Journal of biosocial science. 2003;35(2):263-85. 
15.Deodati A, Cianfarani S. Impact of growth hormone therapy on adult height of children with idiopathic short stature: systematic review. Bmj. 2011;342:c7157.

16.Lin-Su K, Harbison MD, Lekarev O, Vogiatzi MG, New MI. Final adult height in children with congenital adrenal hyperplasia treated with growth hormone. The Journal of clinical endocrinology and metabolism. 2011;96(6):1710-7.

17.Gu Y, Li H, Bao X, Zhang Q, Liu L, Meng G, et al. The Relationship Between Thyroid Function and the Prevalence of Type 2 Diabetes Mellitus in Euthyroid Subjects. The Journal of clinical endocrinology and metabolism. 2017;102(2):434-42.

18.Gao X, Fan JG. Diagnosis and management of non-alcoholic fatty liver disease and related metabolic disorders: consensus statement from the Study Group of Liver and Metabolism, Chinese Society of Endocrinology. Journal of diabetes. 2013;5(4):406-15.

19.Alberti KG, Eckel RH, Grundy SM, Zimmet PZ, Cleeman JI, Donato KA, et al. Harmonizing the metabolic syndrome: a joint interim statement of the International Diabetes Federation Task Force on Epidemiology and Prevention; National Heart, Lung, and Blood Institute; American Heart Association; World Heart Federation; International Atherosclerosis Society; and International Association for the Study of Obesity. Circulation. 2009;120(16):1640-5.

20.Frith J, Day CP, Henderson E, Burt AD, Newton JL. Non-alcoholic fatty liver disease in older people. Gerontology. 2009;55(6):607-13.

21.Foghsgaard S, Andreasen C, Vedtofte L, Andersen ES, Bahne E, Strandberg C, et al. Nonalcoholic Fatty Liver Disease Is Prevalent in Women With Prior Gestational Diabetes Mellitus and Independently Associated With Insulin Resistance and Waist Circumference. Diabetes care. 2017;40(1):109-16.

22.Loomba R, Abraham M, Unalp A, Wilson L, Lavine J, Doo E, et al. Association between diabetes, family history of diabetes, and risk of nonalcoholic steatohepatitis and fibrosis. Hepatology (Baltimore, Md). 2012;56(3):943-51.

23.Ghamar-Chehreh ME, Khedmat $H$, Amini M, Taheri S. Predictive value of having positive family history of cardiovascular disorders, diabetes mellitus, dyslipidemia, and hypertension in non-alcoholic fatty liver disease patients. Acta medica Iranica. 2013;51(5):307-13.

24.Lorenzo C, Williams K, Stern MP, Haffner SM. Height, ethnicity, and the incidence of diabetes: the San Antonio Heart Study. Metabolism: clinical and experimental. 2009;58(11):1530-5.

25.Janghorbani M, Momeni F, Dehghani M. Hip circumference, height and risk of type 2 diabetes: systematic review and meta-analysis. Obesity reviews: an official journal of the International Association for the Study of Obesity. 2012;13(12):1172-81. 
26.Vangipurapu J, Stancakova A, Jauhiainen R, Kuusisto J, Laakso M. Short Adult Stature Predicts Impaired beta-Cell Function, Insulin Resistance, Glycemia, and Type 2 Diabetes in Finnish Men. The Journal of clinical endocrinology and metabolism. 2017;102(2):443-50.

27.Yang C, Yang S, Xu W, Zhang J, Fu W, Feng C. Association between the hyperuricemia and nonalcoholic fatty liver disease risk in a Chinese population: A retrospective cohort study. PloS one. 2017;12(5):e0177249.

28. Takahashi Y. Essential roles of growth hormone (GH) and insulin-like growth factor-I (IGF-I) in the liver. Endocrine journal. 2012;59(11):955-62.

29.Ahrens M, Ammerpohl O, von Schonfels W, Kolarova J, Bens S, Itzel T, et al. DNA methylation analysis in nonalcoholic fatty liver disease suggests distinct disease-specific and remodeling signatures after bariatric surgery. Cell metabolism. 2013;18(2):296-302.

30.Ouni M, Gunes Y, Belot MP, Castell AL, Fradin D, Bougneres P. The IGF1 P2 promoter is an epigenetic QTL for circulating IGF1 and human growth. Clinical epigenetics. 2015;7:22.

31. Holland ML, Lowe R, Caton PW, Gemma C, Carbajosa G, Danson AF, et al. Early-life nutrition modulates the epigenetic state of specific rDNA genetic variants in mice. Science (New York, NY). 2016;353(6298):495-8.

32.Pruis MG, Lendvai A, Bloks VW, Zwier MV, Baller JF, de Bruin A, et al. Maternal western diet primes nonalcoholic fatty liver disease in adult mouse offspring. Acta physiologica (Oxford, England). 2014;210(1):215-27.

33.Tyrrell J, Jones SE, Beaumont R, Astley CM, Lovell R, Yaghootkar H, et al. Height, body mass index, and socioeconomic status: mendelian randomisation study in UK Biobank. Bmj. 2016;352:i582.

34.Kennedy BP, Kawachi I, Glass R, Prothrow-Stith D. Income distribution, socioeconomic status, and self rated health in the United States: multilevel analysis. Bmj. 1998;317(7163):917-21.

35.Saadeh S, Younossi ZM, Remer EM, Gramlich T, Ong JP, Hurley M, et al. The utility of radiological imaging in nonalcoholic fatty liver disease. Gastroenterology. 2002;123(3):745-50.

\section{Tables}


Table 1. Baseline characteristics of participants by NAFLD status ${ }^{a}$.

\begin{tabular}{|c|c|c|c|}
\hline & \multicolumn{2}{|c|}{ NAFLD } & \multirow[t]{2}{*}{$P$ value ${ }^{\mathrm{b}}$} \\
\hline & No & Yes & \\
\hline No. of subjects & 29,749 & 6,245 & \\
\hline Age (y) ${ }^{c}$ & $38.3(38.2,38.4)$ & $41.4(41.1,41.7)$ & $<0.0001$ \\
\hline Sex (males, \%) & 36.9 & 62.1 & $<0.0001$ \\
\hline BMI $\left(\mathrm{kg} / \mathrm{m}^{2}\right)$ & $22.2(22.2,22.3)$ & $24.9(24.8,25.0)$ & $<0.0001$ \\
\hline Waist circumference (cm) & $75.4(75.4,75.5)$ & $83.4(83.1,83.6)$ & $<0.0001$ \\
\hline Metabolic syndromes (yes, \%) & 8.3 & 26.6 & $<0.0001$ \\
\hline TC & $4.62(4.62,4.63)$ & $4.83(4.81,4.86)$ & $<0.0001$ \\
\hline LDL & $2.65(2.65,2.66)$ & $2.90(2.88,2.92)$ & $<0.0001$ \\
\hline TG & $0.87(0.87,0.88)$ & $1.24(1.22,1.25)$ & $<0.0001$ \\
\hline HDL & $1.48(1.48,1.48)$ & $1.28(1.27,1.28)$ & $<0.0001$ \\
\hline FBG & $4.78(4.78,4.78)$ & $4.89(4.88,4.91)$ & $<0.0001$ \\
\hline SBP & $114.6(114.6,114.8)$ & $120.4(120.1,120.8)$ & $<0.0001$ \\
\hline DBP & $72.1(72.1,72.2)$ & $76.1(75.9,76.4)$ & $<0.0001$ \\
\hline ALT (U/L) & $15.2(15.2,15.3)$ & $20.1(19.8,20.3)$ & $<0.0001$ \\
\hline Smoking status (\%) & & & - \\
\hline Smoker & 14.3 & 26.6 & $<0.0001$ \\
\hline Ex-smoker & 2.0 & 4.3 & $<0.0001$ \\
\hline Non-smoker & 83.7 & 69.2 & $<0.0001$ \\
\hline \multicolumn{4}{|l|}{ Drinker status (\%) } \\
\hline Everyday & 1.6 & 2.4 & $<0.0001$ \\
\hline Sometime & 37.3 & 47.4 & $<0.0001$ \\
\hline Ex-drinker & 4.2 & 4.5 & 0.27 \\
\hline Non-drinker & 56.9 & 45.7 & $<0.0001$ \\
\hline
\end{tabular}

Family history of diseases (\%) 


\begin{tabular}{lccc} 
CVD & 28.1 & 34.8 & $<0.0001$ \\
\hline Hypertension & 48.0 & 55.3 & $<0.0001$ \\
\hline Hyperlipidemia & 0.5 & 0.6 & 0.055 \\
\hline Diabetes & 20.7 & 26.0 & $<0.0001$ \\
\hline
\end{tabular}

a NAFLD, non-alcoholic fatty liver disease; BMI, body mass index; MS, metabolic syndromes; TC, total cholesterol; LDL, low-density lipoprotein cholesterol; TG, triglycerides; HDL, high-density lipoprotein cholesterol; FBG, fasting blood glucose; SBP, systolic blood pressure; DBP, diastolic blood pressure; ALT, alanine aminotransferase; CVD, cardiovascular disease.

$\mathrm{b}$ Analysis of variance or logistic regression analysis.

C Geometric mean (95\% confidence interval) (all such values). 
Table 2. Cohort analysis: adjusted associations of height quintiles with NAFLD ${ }^{a}$ in males.

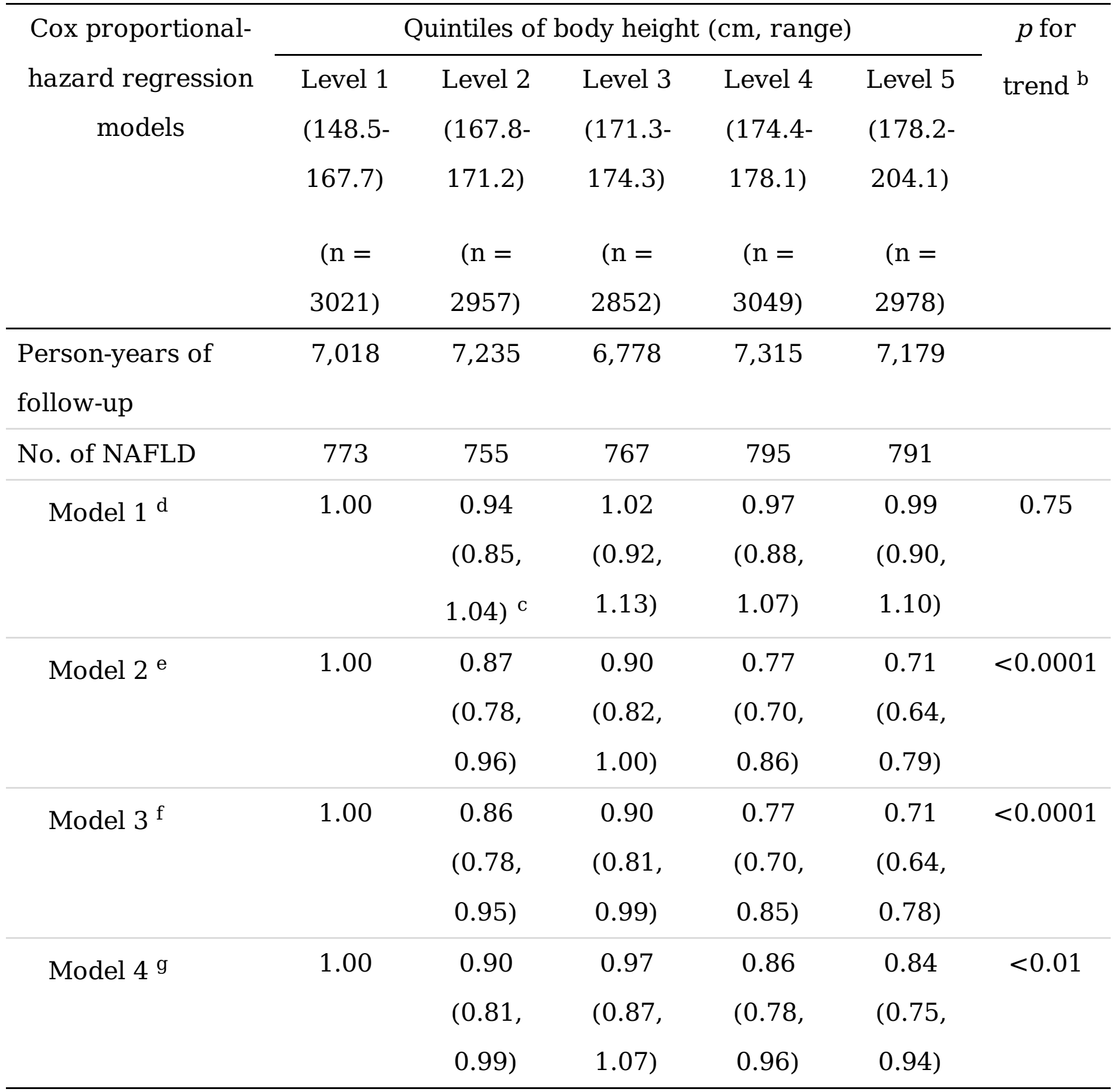

a NAFLD, non-alcoholic fatty liver disease.

b Analysis by Cox proportional hazards model.

C Adjusted hazard ratios (95\% confidence interval) (all such values).

d Crude

e Adjusted for age and waist circumference. 
f Adjusted for age, waist circumference, smoking status, drinking status, metabolic syndrome, and family history of cardiovascular disease, hypertension, hyperlipidemia, and diabetes.

g Adjusted for age, body mass index, waist circumference, smoking status, drinking status, metabolic syndrome, and family history of cardiovascular disease, hypertension, hyperlipidemia, and diabetes. 
Table 3. Cohort analysis: adjusted associations of height quintiles with NAFLD ${ }^{a}$ in females.

\begin{tabular}{|c|c|c|c|c|c|c|}
\hline \multirow{6}{*}{$\begin{array}{c}\text { Cox proportional- } \\
\text { hazard regression } \\
\text { models }\end{array}$} & \multicolumn{5}{|c|}{ Quintiles of body height (cm, range) } & \multirow{6}{*}{$\begin{array}{l}p \text { for } \\
\text { trend }{ }^{b}\end{array}$} \\
\hline & Level 1 & Level 2 & Level 3 & Level 4 & Level 5 & \\
\hline & (138.0- & (156.3- & (159.6- & (162.2- & (165.5- & \\
\hline & 156.2) & 159.5) & 162.1) & 165.4) & 184.6) & \\
\hline & $(\mathrm{n}=$ & $(\mathrm{n}=$ & $(\mathrm{n}=$ & $(\mathrm{n}=$ & $(\mathrm{n}=$ & \\
\hline & 4223) & 4442) & 3947) & 4145) & 4370) & \\
\hline \multirow{2}{*}{$\begin{array}{l}\text { Person-years of } \\
\text { follow-up }\end{array}$} & 11,318 & 12,593 & 11,153 & 11,576 & 12,736 & \\
\hline & & & & & & \\
\hline No. of NAFLD & 548 & 543 & 454 & 416 & 404 & \\
\hline \multirow[t]{3}{*}{ Model $1 \mathrm{~d}$} & 1.00 & 0.88 & 0.83 & 0.73 & 0.64 & $<0.0001$ \\
\hline & & $(0.78$ & (0.73, & $(0.64$ & (0.57, & \\
\hline & & $0.99)^{\mathrm{C}}$ & $0.94)$ & 0.83) & $0.73)$ & \\
\hline \multirow[t]{3}{*}{ Model 2 e } & 1.00 & 0.91 & 0.85 & 0.75 & 0.62 & $<0.0001$ \\
\hline & & (0.81, & $(0.75$ & (0.66, & $(0.55$ & \\
\hline & & 1.02) & 0.96) & 0.85) & $0.71)$ & \\
\hline \multirow[t]{3}{*}{ Model $3^{f}$} & 1.00 & 0.92 & 0.85 & 0.78 & 0.65 & $<0.0001$ \\
\hline & & $(0.82$, & $(0.75$ & $(0.68$, & $(0.57$ & \\
\hline & & $1.04)$ & 0.97) & 0.89) & $0.74)$ & \\
\hline \multirow[t]{3}{*}{ Model 49} & 1.00 & 0.97 & 0.98 & 0.93 & 0.84 & 0.02 \\
\hline & & (0.86, & $(0.86$ & $(0.81$, & $(0.73$ & \\
\hline & & 1.09) & 1.11) & 1.06) & $0.96)$ & \\
\hline
\end{tabular}

a NAFLD, non-alcoholic fatty liver disease.

b Analysis by Cox proportional hazards model.

C Adjusted hazard ratios (95\% confidence interval) (all such values). 
d Crude

e Adjusted for age and waist circumference.

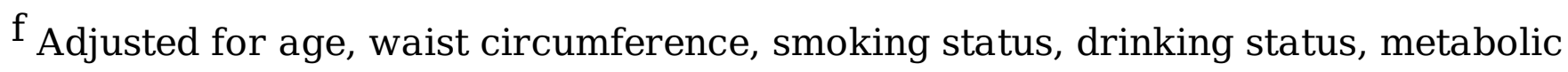
syndrome, and family history of cardiovascular disease, hypertension, hyperlipidemia, and diabetes.

g Adjusted for age, body mass index, waist circumference, smoking status, drinking status, metabolic syndrome, and family history of cardiovascular disease, hypertension, hyperlipidemia, and diabetes. 